\title{
Parallel Machine Production and Transportation Operations' Scheduling with Tight Time Windows
}

\author{
Yang Jiang $\mathbb{D}^{1},{ }^{1}$ Tong He, ${ }^{1}$ Jie Xiong, ${ }^{2}$ Xin $W u,{ }^{3}$ and Yao Chen ${ }^{4}$ \\ ${ }^{1}$ School of Management, Shenyang University of Technology, Shenyang 110870, China \\ ${ }^{2}$ Beijing Key Laboratory of Traffic Engineering, Beijing University of Technology, Beijing 100124, China \\ ${ }^{3}$ School of Sustainable Engineering and the Built Environment, Arizona State University, Tempe 85281, Arizona, USA \\ ${ }^{4}$ Department of Industrial Systems Engineering and Management, National University of Singapore, 119077, Singapore \\ Correspondence should be addressed to Yang Jiang; jiangyang0121@163.com
}

Received 6 February 2020; Revised 29 July 2020; Accepted 23 September 2020; Published 10 October 2020

Academic Editor: Lingzhong Guo

Copyright $\odot 2020$ Yang Jiang et al. This is an open access article distributed under the Creative Commons Attribution License, which permits unrestricted use, distribution, and reproduction in any medium, provided the original work is properly cited.

\begin{abstract}
This study addresses a parallel machine production and transportation operations' scheduling problem with a tight time window associated with the transfer and delivery process. The orders located at the parallel machines need to be delivered to customers by train. Each order must be processed within a limited completion time in order for the product to be matched with the optimal trip to its destination within the delivery period. A mathematical analysis method is used to reveal the impact of tight time windows on the scheduling of production and transportation operations. The order transfer redundancy time and order transfer waiting time are employed to reflect the impact scheduling of production on the transfer process. The order delivery redundancy time and order delivery waiting time are used to describe delivery operations. The goal is to maximize the coordination level of order transfer and delivery, which are reflected in the order transfer time and the order delivery time, respectively. Additionally, a simulated annealing algorithm using the column generation technique was developed to solve this problem. The results show that the use of the system coordination model in this method obviously improves the number of successful transfers and deliveries.
\end{abstract}

\section{Introduction}

Made-to-order or time-sensitive goods are a hot topic that has been addressed by many researchers (Shu et al. [1] and Federgruen et al. [2]), and completed orders are usually required to be delivered to customers within tight time windows. It is believed that benefits are anticipated for integrating production scheduling with transportation operations [3-5]. The sharing of manufacturing resources means that raw materials and semifinished products located at different suppliers and customers are transferred widely and frequently $[6,7]$.

The scheduling of parallel batch production adding transportation operations should integrate tight time windows associated with rail transport plans to ensure that timesensitive orders are met. The integrated scheduling problem features several special characteristics. Compared with a normal distribution system design problem, the time-critical aspect means that the longest lead-time from a production line to any accessible trip's destination is subject to timedeadline restrictions that limit the order completion time. Furthermore, the orders should be transferred onto the selected trips within tight time windows imposed by the rail transport plan (Goossens et al. [8] and Fu et al. [9]). The challenges of rail time windows for time-sensitive products can be inferred according to Kang et al. [10] and Wu et al. [11]. Meanwhile, a trip can ship multiple orders to multiple destinations along the same railway line, which enriches the diversity of production schedules and trip selection (DiazMadronero et al. [12] and Cao et al. [13]).

With the development of off-site production businesses, it is increasingly urgent to coordinate the activities of both production and transportation operations to facilitate the dispatch and receipt of goods. The contributions of this paper are summarized as follows. First, a mathematical formula is developed to describe the relationships between 
the order transfer redundancy time (OTR), order transfer waiting time (OTW), order delivery redundancy time (ODR), and order delivery waiting time (ODW). The comprehensive formula can be used to improve the connecting relationship between the production line and trains. Meanwhile, the formula is also used to distinguish between the last feasible connecting train and the nonlast ones and to judge whether the feasible connecting trains will be missed. Moreover, a parallel machine production and distribution model is proposed to optimize transfer and delivery considering tight time windows. The complexity of the problem is based primarily on the use of a large set of discrete variables. A simulated annealing algorithm combined with a column generation approach (SACG) is designed to address the numerical testing network model. The results of the numerical example show that the quantitative performances of the delivery timeliness improved by the connecting quality (OT) are much better than those from the view of the delivery time window (OD).

In the remainder of this study, we first address some synergetic indexes first to measure the effect of tight time windows on production and transportation operations in Sections 3.1 and 3.2. A mathematical method is employed to reveal the relationships between OTR and OTW, and a similar scenario analysis approach is also applied to ODR and ODW for transportation operations. Section 3.3 develops a coordination scheduling model of parallel machine production and transportation operations to maximize the coordination level of order transfer and delivery, which reflects OT and OD. A simulated annealing algorithm using the column generation technique was developed, and we conduct a case study in Section 4. Finally, we conclude this study in Section 5 with discussions on possible extensions.

\section{Literature Review}

Research on the integration of scheduling models of production and distribution, which is known as integrated production-distribution planning or scheduling, has been relatively recent and includes studies such as Pundoor and Chen [5], Pornsing et al. [14], Zhong and Jiang [15], Russel et al. [16], Kishimoto et al. [17], and Ma et al. [18]. Many made-to-order or time-sensitive products are often needed to be delivered to their customers in time-critical modes in which tasks must be executed within a tight time frame. Most extant studies consider only the determination of location decisions or production scheduling through the application of mixed-integer programming (MIP) models (Chen [3]). Their objective functions are to minimize the total operating cost, service time, or budget input [19-27]. These models are subjected to production capacity, fleet size, job processing, or batching constraints, e.g., Cheng et al. [28], Devapriya et al. [29], and Noroozi et al. [30]. For example, Hajiaghaei-Keshteli et al. [21] formulated a mathematical model to study the production and transportation system and capacity and the cost of rail transportation, which were the focus of their article. Jiang et al. [31] applied a scenario analysis method to establish performance measures to minimize the total waiting time. One of the tasks of this paper is to handle tight time windows and limit the deadline-dependent lead time within a given time window. The time deadlines in the transfer and delivery process that are imposed to guarantee on-time delivery have an impact on parallel batch scheduling and transfer trip selection. In particular, parallel batch scheduling complicates the model construction and solution.

Parallel batch scheduling problems under the influence of rail timetables are the focus of our research. In previous studies related to production and distribution system design problems, trucks and planes were used to serve customers as an important transport mode, and mathematical models were proposed to optimize the production and road and air transportation coordination problem (Chang and Lee [20], Chen and Vairaktarakis [4], Zhong and Jiang [15], Moons et al. [32], Wang and Cheng [33], Xuan [34], Zhong et al. [35], Seyedhosseini and Ghoreyshi [36], Gong et al. [37], Zandieh and MollaAlizadeh-Zavardehi [38], and Delavar et al. [39]). For instance, an integrated production and distribution scheduling problem was considered by Devapriya et al. [29], and in this research, the trucks' routes and fleet size are the important decisions to be made. Azadian et al. [40] researched the order contract producing problem from a manager's perspective and proposed an integrated scheduling model on the coordination of production and transportation planning. It is not difficult to see that this class of problems employed a vehicle routing problem to satisfy its delivery shipments. The coordination between production and rail transportation in an operational time dimension is one of the less focused-upon aspects and is our major task and focus. The mode of railway transportation is quite different from that of air transportation in transport plans and rolling stock (Pemberton [41]; Ho and Leung [38]). The distribution system design integrates the knowledge of path selection, and the time window caused by the rail timetable makes our focus more novel and interesting. To the best of our knowledge, few studies have investigated these topics in the context of railway timetables.

In conclusion, our method not only takes relevant accessibility into account but also takes delivery timeliness as a time window constraint into account. Of course, this approach is common in other articles. However, more importantly, not only delivery timeliness (customer service levels at the individual order level) is guaranteed by the tight delivery time window but a comprehensive formula is also introduced to measure delivery timeliness or customer service levels at the individual order level. The comprehensive formula can be used to distinguish between the last feasible connecting train and the nonlast ones and to judge whether the feasible connecting trains will be missed. Meanwhile, the formula is employed to improve the connecting relationship between the production line and trains. This formula is summarized in the scenario analysis introduced in our research. 


\section{Integrated Scheduling Coordination Model}

3.1. Order Transfer Redundant Time and Order Transfer Waiting Time. The OTR describes whether the transfer relationship can be established successfully. If OTR is equal to or greater than 0 , it means that the transfer can be carried out, as shown in scenarios (a) and (b) in Figure 1. However, the order may miss all the feasible trips to its destination when OTR is less than 0 , as shown in scenario (c) in Figure 1.

The OTW is the waiting time for order $i$ when it transfers from the production line $l$ to its selected connecting trip $l^{\prime}$. As shown in Figure 1, the OTW is forced to be $M$ when order $i$ misses all the feasible trips, where Mrepresents an infinite value.

The OTR and OTW can be calculated by the following equation:

$$
\left\{\begin{array}{l}
t_{\mathrm{sill}}^{r}=t_{\mathrm{sl}}^{D}-t_{\mathrm{sil}}^{A}-t_{\mathrm{sll}}^{\mathrm{Tra}} \\
t_{\mathrm{sill}}^{w}=y_{\mathrm{il}} \cdot t_{\mathrm{sl}^{\prime}}^{D^{\prime}}-t_{\mathrm{sil}}^{A}-t_{\mathrm{sll}}^{\mathrm{Tra}}+\left(1-y_{\mathrm{il}^{\prime}}\right) \cdot M .
\end{array}\right.
$$

Furthermore, some interconnections between OTR and OTW can be found in Figure 1. Based on the three different relationships between the order completion time and feasible trip departure time, OT is introduced in equation (2). In each scenario, two timelines are included: production and transportation. To facilitate modeling, $\theta$ is a fixed value that represents the penalty value for missing feasible connecting trips.

$$
t_{\text {sill' }}^{h}=\left\{\begin{array}{l}
t_{\text {sill' }}^{r}-t_{\text {sill' }}^{w}=t_{\mathrm{sl}^{\prime}}^{D}-t_{\mathrm{sl}^{\prime}}^{D^{\prime}} \geq 0, \text { when } t_{\text {sill' }}^{r}-t_{\text {sill' }}^{w} \geq 0 \\
t_{\mathrm{sill}^{\prime}}^{r}-t_{\mathrm{sill}}^{w}=-\theta, \text { when } t_{\mathrm{s} l^{\prime}}^{D}-\left(t_{\mathrm{sil}}^{A}+t_{\mathrm{sll}}^{\mathrm{Tra}}\right)<0
\end{array}\right.
$$

\subsection{Order Delivery Redundant Time and Order Delivery} Waiting Time. The ODR is a measure of delivery success. If the ODR is greater than 0 , the delivery is successful, as shown in scenarios (a) and (b) of Figure 2. However, the order may violate the tight time window when the ODR is less than 0 , as shown in scenario (c) of Figure 2.

The ODW is the waiting time for order $i$ before the delivery is allowed by the tight time window. As shown in Figure 2, the ODW is forced to be $M$ when order $i$ violates the tight time window, where Mrepresents an infinite value.

The ODR and ODW are formulated in the following equation:

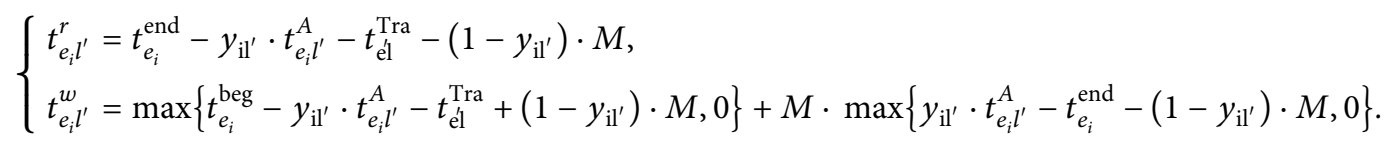

Similarly, some interconnections between the ODR and ODW can be found in Figure 2. Based on the three different relationships of the selected trip arrival time and the tight time window, OD is introduced in equation (4). In each scenario, two timelines are included: production and transportation. To facilitate modeling, $\alpha, \mu$, and $\delta$ are fixed values that present the penalty values for violating the tight time window and $|\delta| \gg|\alpha|$.

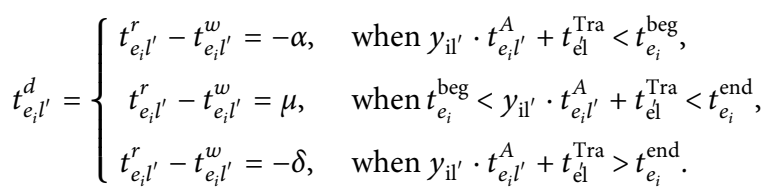

3.3. Model Formulation. The objective function includes two aspects. On the one hand, our research aims to maximize OTR and ODR. This means that the improvement of transfer success and delivery success is a measure of scheduling. On the other hand, minimizing OTW and ODW is a measure of system cooperative efficiency. Therefore, the objective function is to maximize OT and OD as follows, where $t_{\text {sill' }}^{h}$ and $t_{e_{i} l^{\prime}}^{d}$ are expressed in equations (2) and (4), respectively:

$$
\operatorname{Max} T=\sum_{s \in S} \sum_{i \in I} \sum_{l \in L} \sum_{l^{\prime} \in L^{\prime}} t_{\text {sill' }}^{h}+\sum_{e_{i} \in E} \sum_{l^{\prime} \in L^{\prime}} t_{e_{i} l^{\prime}}^{d} .
$$

(1) For any production line $l \in L$, if $i$ and $j$ are processed continuously, then the order completion time is formulated in (6); if $i$ is the first order on $l$, then see equation (7).

$$
\begin{aligned}
t_{\mathrm{sjl}}^{A}+p_{i} Q_{i} & =t_{\mathrm{sil}}^{A} \text {, when } \sum_{j}^{k} \sum_{i \neq j}^{k} r_{j i}^{l}=1, \quad \forall l \in L, \\
t_{\mathrm{sil}}^{A} & =p_{i} Q_{i}, \text { when } \sum_{j}^{k} \sum_{i \neq j}^{k} r_{j i}^{l}=0, \quad \forall l \in L .
\end{aligned}
$$

(2) Constraints imposed by integer programming:

$$
\begin{aligned}
& \sum_{l} \sum_{i} \sum_{j \neq i} r_{i j}^{l}=1, \\
& r_{i j}^{l}=\{0,1\}, \quad \forall i, j \in I, l \in L=1, \\
& y_{i l^{\prime}}=\{0,1\}, \quad \forall i \in I, l^{\prime} \in L^{\prime} .
\end{aligned}
$$

(3) The total number of processing steps should not exceed the capacity of each production line. 

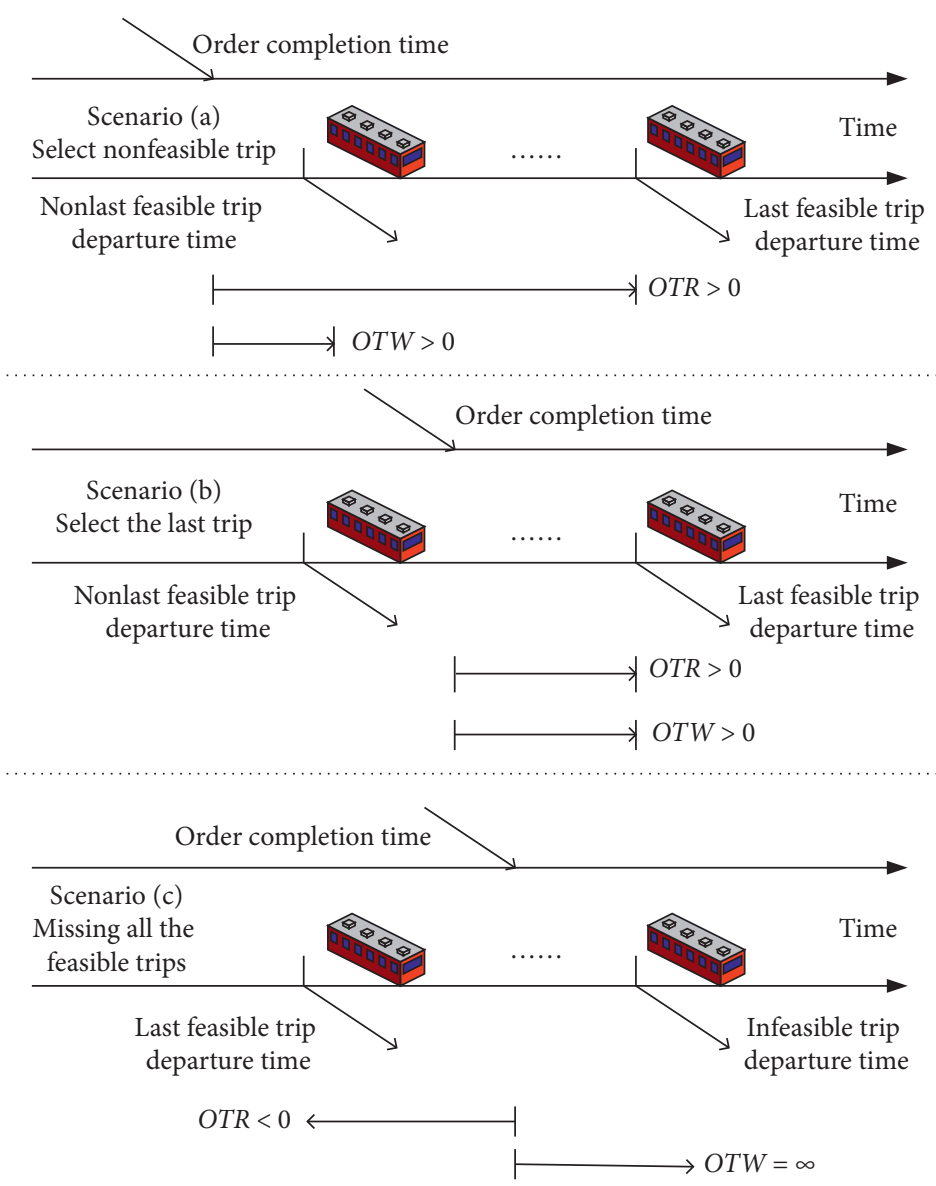

Figure 1: Three different relationships between the order completion time and feasible trip departure time.

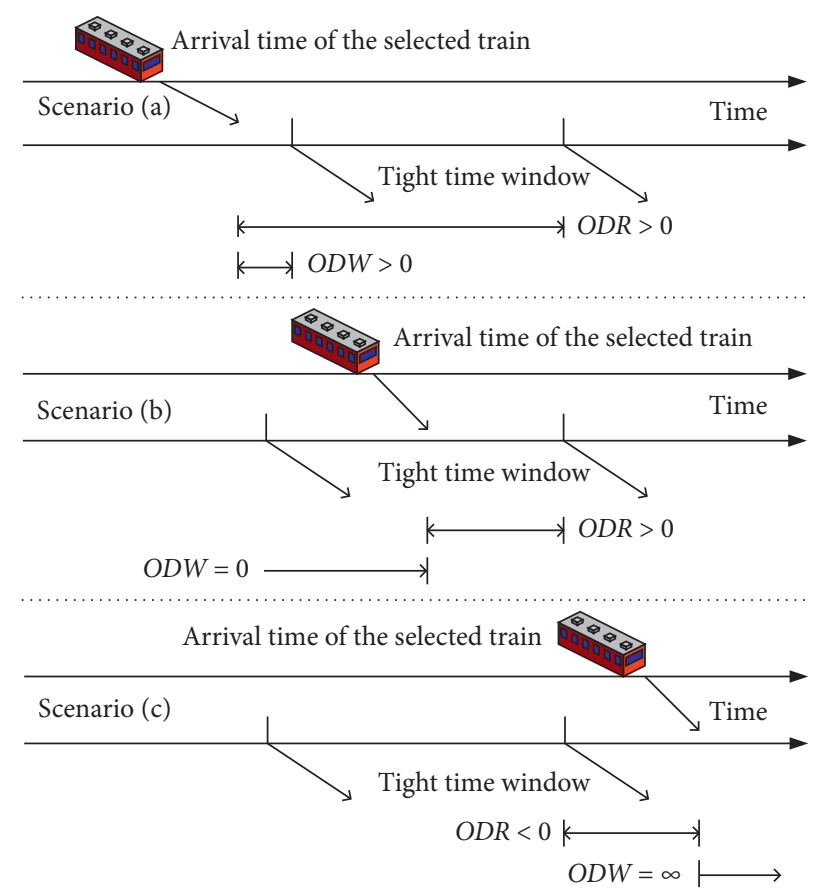

Figure 2: Three different relationships between the order arrival time and the tight time window. 


$$
\sum_{l \in L} \sum_{i \in I} y_{i l} \cdot Q_{i} \leq \operatorname{Cap}_{l} \quad \forall l .
$$

\section{Solution Algorithm and Case Study}

4.1. Simulated Annealing Embedded in the Column Generation Approach. Integration problems such as productiondistribution and location-routing problems belong to the NP-hard class, so it is difficult to solve them with exact algorithms (Perl and Daskin [42]). Heuristic approaches have become a more feasible algorithm (Nagy and Salhi [43]). For example, Jiang et al. [31] used a genetic algorithm to handle supply chain management with a single machine and transportation scheduling problem. Additionally, the simulated annealing algorithm [44] and cross-entropy method [11] are effective and have been applied to solve this kind of problem.

Simulated annealing (SA) has been proven by many researchers to be an effective method for solving combinatorial optimization problems. Its efficiency and effectiveness in solving a variety of real-world issues, e.g., SA combined with local search for solving vehicle routing problems with time windows (Lin et al. [45]), flow path and location problems (Hamzeei et al. [46]), timetabling problems (Daduna and Vo [47]), network design optimization (Friesz et al. [48]), and distribution center problems (Wei and Zhou. [49]), have been proven. Meanwhile, Ahin and Türkbey [50] presented that the approximate Pareto optimal sets we have found include almost all the previously obtained results and many more approximate Pareto optimal solutions. The results indicated that SA can determine the best solution most times. Therefore, we have a reason to believe that our proposed algorithm is effective and can be applied to solve our problem.

Column generation (CG) is an effective method to solve large-scale linear programming problems, especially for large-scale models. The CG technique has been widely used to solve a variety of real-world issues, e.g., crew scheduling (Soumis [51]), production scheduling (Chen and Powell [52]), vehicle routing (Skitt and Levary [53]), air transport (Liang et al. [54]), and issues in the medical and healthrelated industries (Wang et al. [55]). The detailed branch and price flow chart is shown in Figure 3. In our problem, CG is employed to obtain the parallel batch scheduling solution. The framework of the developed SACG in our research is shown in Figure 4.

The encoding style of solutions in Figure 5 is a matrix representation. As shown, rows indicate the production line set, and the columns indicate the order set. We used blank cells to fill in some rows since the number of orders in each production line may not be equal. Each order cell must be covered in a certain production line, and the number of cells is equal to the total number of orders.

After generating an initial solution, replacing moves are performed to search the alternative set. When a dropping move is called, it tries to select the trip. However, due to the condition of the production schedule, it may fail to find a transfer trip or deliver successfully, which may cause the infeasibility of transfer or delivery. The cooling function defines the temperature $T_{i}$ for each step of the algorithm $i$. It has a strong impact on the success of the SA algorithm. In the proposed algorithm, the linear strategy for updating the temperature is selected.

$$
T_{i}=\text { Initial_Temperature }-(i \times \text { Cooling_Rate }),
$$

where $T_{i}$ represents the temperature at iteration $i$ and Cooling_Rate and Initial_Temperature are the specified constant value and the initial temperature, respectively.

Last, in the proposed algorithm, the number of iterations and the number of reduced temperatures with no improvement are used as stopping criteria.

4.2. Numerical Test. Figure 6 is an example network including multiple production lines, six railway stations, ten orders, and eight railway trips. The starting point of all trips is considered as the location of the production facility. The timetable of all trips is shown in Table 1. The second column indicates the departure time of each trip. Nonzero elements in columns 3 to 8 of Table 1 characterize the path of each trip, and each element indicates the arrival time of the trip at the corresponding station. If the path of a trip covers the destination of an order and the order completion time is before the trip departs, it is a feasible solution that the order selects the trip.

4.2.1. Network Solution. We test the model and SACG algorithm on a personal computer with Intel Core i5, $2.60 \mathrm{GHz} \mathrm{CPU}$, and $4 \mathrm{~GB}$ RAM. We set the initial temperature to 500 , the descent rate to 0.95 , and the terminal condition to 0.1 . Let $\theta=100, \alpha=10, \mu=10$, and $\delta=100$. The cost of the parallel production line is defined as 0 at first, and it will be deeply discussed in the subsequent analysis. The SACG can solve the model in a very short time, and the detailed results are shown in Table 2. The OTW, $t_{\text {sill }}^{w}$, is the key indicator to measure the production success connection. It shows that $100 \%$ of orders achieve successful transfer in the sample network. Meanwhile, ODW is used to judge whether the delivery is successful. From Table 2, we can see that orders 1 and 6 fail to deliver. We obtain the same performance as the experiment in Jiang et al. [31], and the number of successful deliveries is 8 . The success rate of order transfer is much better than that in Jiang et al. [31].

4.2.2. Algorithm Performance. The iteration trace of SACG is shown in Figure 7. It can be seen that the genetic algorithm finds the optimal solution in a very short time. The iteration becomes stable after 10 iterations, and the optimal solution with the objective function is -99.5 . The detailed results are presented in the first row of Table 3. Compared with the solution given in [31], the optimized solution we found decreases the total cost by nearly $50 \%$.

We adopt a genetic algorithm to solve the numerical network again and compare the performance with SA. The results of all algorithms are similar, and the correctness of the algorithm is verified (Table 3 ). 


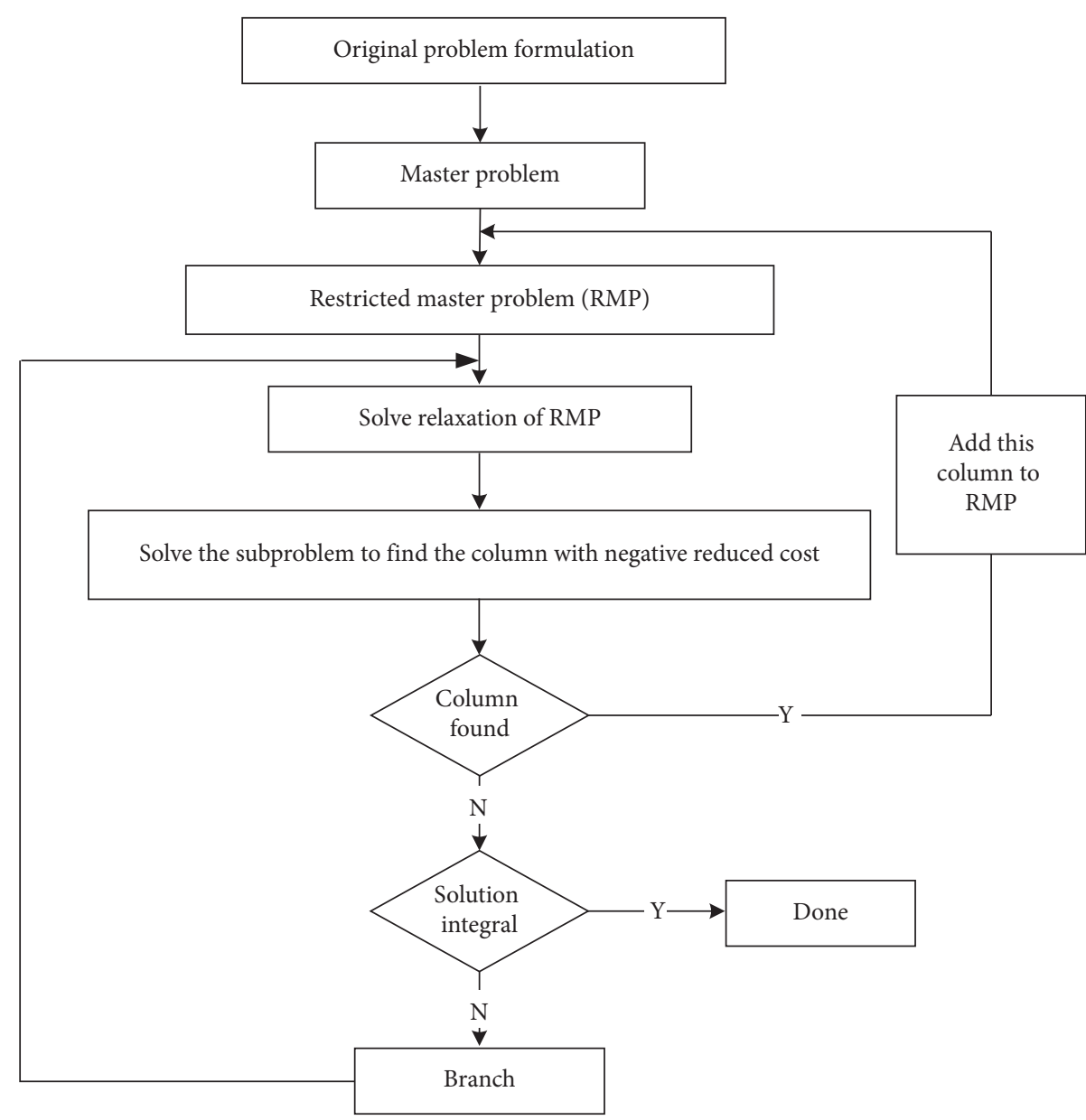

FIgURE 3: The branch and price process.

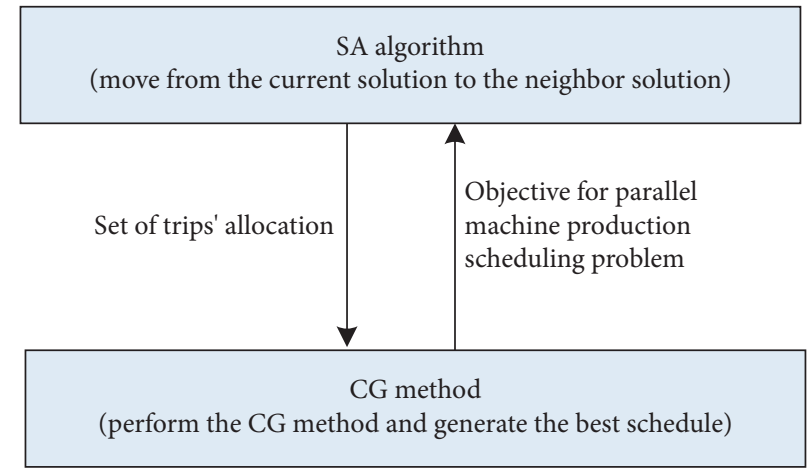

FIgURE 4: The framework of the developed SACG.

Two major algorithm parameters related to iteration times are tested here, and the results are shown in Table 4. Different combinations of $T_{0}$ and qare used to solve the problem. The results show that when we set a larger initial temperature $\left(T_{0}\right)$ and a larger descent rate $(q)$, the optimal result can be improved to a certain extent. When $T_{0}=0$ or $q=0$, the SACG algorithm cannot work.
4.2.3. Comparison of Different Goals. To verify the proposed model (Max OT + OD), we compare the other two objectives, i.e., Max OT and Max OD. The experiment is completed under the same conditions and with the same data. Table 5 lists the details of the results.

There are ten successful transfers and seven successful deliveries when maximizing OT. Meanwhile, maximizing OD can obtain eight successful transfers and eight successful 


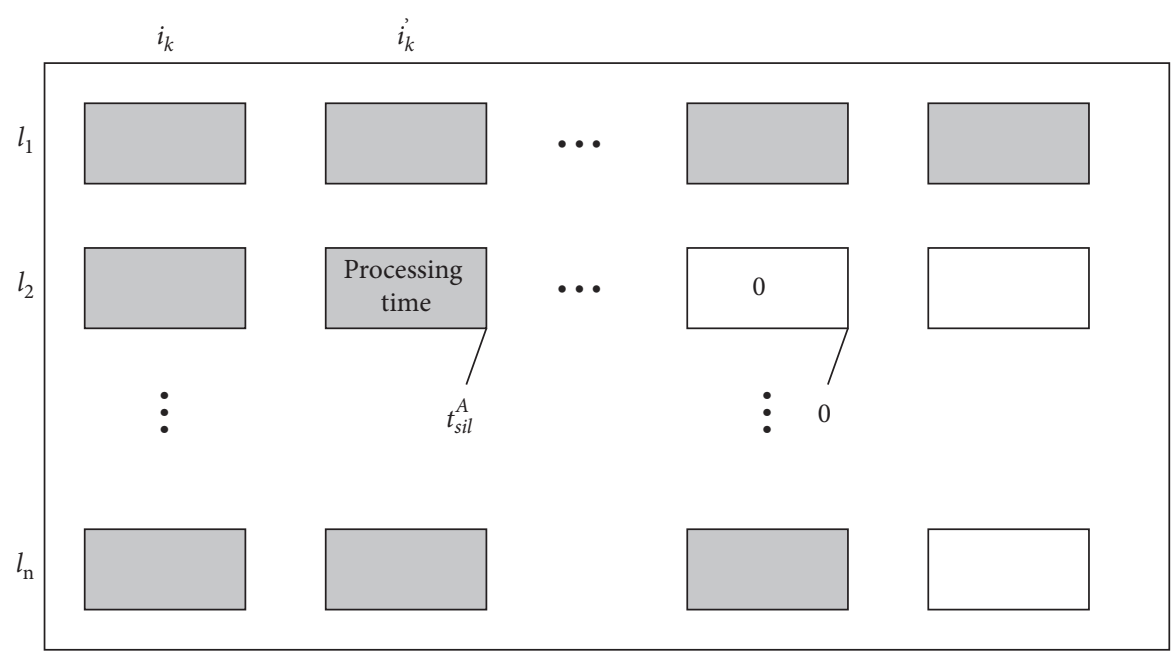

Figure 5: The encoding style and solution expression.

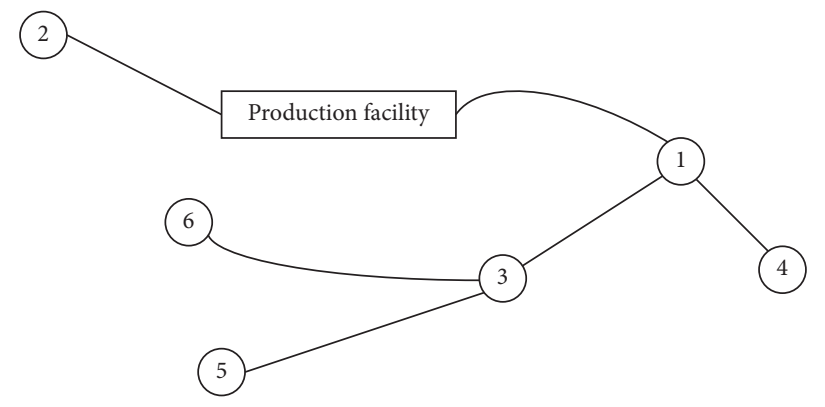

\begin{tabular}{|c|c|c|c|c|c|c|c|c|c|c|}
\hline Order & 1 & 2 & 3 & 4 & 5 & 6 & 7 & 8 & 9 & 10 \\
\hline Destination & 3 & 2 & 4 & 2 & 1 & 5 & 4 & 6 & 1 & 5 \\
\hline
\end{tabular}

\begin{tabular}{|c|c|c|c|c|c|c|c|c|}
\hline Trip & 1 & 2 & 3 & 4 & 5 & 6 & 7 & 8 \\
\hline Destination & 5 & 2 & 6 & 5 & 4 & 5 & 2 & 4 \\
\hline
\end{tabular}

FIgURE 6: Illustration of a sample network.

TABle 1: Timetable of the sample network.

\begin{tabular}{|c|c|c|c|c|c|c|c|}
\hline Train line & Departure time & 1 & 2 & 3 & 4 & 5 & 6 \\
\hline 1 & 1 & 3.5 & -- & 5 & - & 7.5 & $\overline{-}$ \\
\hline 2 & 1.5 & - & 4.5 & - & - & - & - \\
\hline 3 & 2 & 3 & - & 4.5 & - & - & 6 \\
\hline 4 & 2.5 & 4.5 & - & 5.5 & - & 8 & - \\
\hline 5 & 3 & 5 & - & - & 6.5 & - & - \\
\hline 6 & 3.5 & 4.5 & - & 6.5 & - & 7.5 & - \\
\hline 7 & 4 & - & 5.5 & - & - & - & - \\
\hline 8 & 4.5 & 5.5 & - & - & 7 & - & - \\
\hline
\end{tabular}

deliveries. Compared with Max OT and Max OD, Max $(\mathrm{OT}+\mathrm{OD})$ achieves an obvious improvement in the objective function.

4.2.4. Analysis of Related Parameters. In this section, we test the influence of relevant parameters on the scheduling model. First, we set the parallel production line cost as $-\infty$ and the capacity of each parallel production line as $\infty$. The result shows that only one production line is needed. The function value is -209 , the number of successful transfers is 9 , and the number of successful deliveries is 8 . We obtained the same optimal solutions as in [31]. Second, we keep other conditions unchanged and test the sensitivity of capacity values when the parallel production line cost is 0 . The details are shown in Table 6, and the trend draws the conclusion that the number of successful transfers is 10 , and the number of successful deliveries is 8 . We can seek some explanation 
TABLE 2: Solution for the sample network.

\begin{tabular}{|c|c|c|c|c|c|c|c|c|c|c|}
\hline Processing time/quality & $0.1 / 1$ & $0.3 / 1$ & $0.4 / 2$ & $0.1 / 3$ & $0.1 / 5$ & $0.1 / 3$ & $0.4 / 1$ & $0.2 / 1$ & $0.2 / 1$ & $0.2 / 4$ \\
\hline Delivery time window & {$[1,7]$} & {$[1,10]$} & {$[1,9]$} & {$[1,10]$} & {$[1,8]$} & {$[1,5]$} & {$[1,6]$} & {$[1,6]$} & {$[1,8]$} & {$[1,4]$} \\
\hline Production scheduling and train allocated & 6 & 10 & 9 & 7 & 3 & 1 & 4 & 2 & 8 & 5 \\
\hline 1 & $\sqrt{ }$ & $\sqrt{ }$ & & & & $\sqrt{ }$ & & & & \\
\hline 2 & & & & & & & $\sqrt{ }$ & $\sqrt{ }$ & & \\
\hline $\begin{array}{l}3 \\
4\end{array}$ & & & $\sqrt{ }$ & & & & & & $\sqrt{ }$ & $\sqrt{ }$ \\
\hline $\begin{array}{l}4 \\
5\end{array}$ & & & & $\sqrt{ }$ & $\sqrt{ }$ & & & & & \\
\hline 6 & & & & & & & & & & \\
\hline \multirow{2}{*}{\multicolumn{11}{|c|}{$\begin{array}{l}7 \\
8\end{array}$}} \\
\hline & & & & & & & & & & \\
\hline$t_{\text {sill }}^{r}$ & 2.9 & 2.6 & 2.8 & 2.5 & 2.0 & 2.7 & 2.8 & 2.6 & 0.1 & 3.2 \\
\hline$t_{\mathrm{sit}}^{\mathrm{su}}$ & 0.4 & 0.1 & 0.3 & 1.0 & 0.5 & 0.2 & 0.3 & 0.1 & 0.1 & 0.7 \\
\hline thill & -1.5 & 1.5 & 5.0 & 2.5 & 0.5 & -1 & 0.5 & 0.5 & 1.0 & 0 \\
\hline 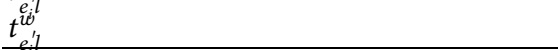 & 0 & 0 & 0 & 0 & 0 & 0 & 0 & 0 & 0 & 0 \\
\hline
\end{tabular}

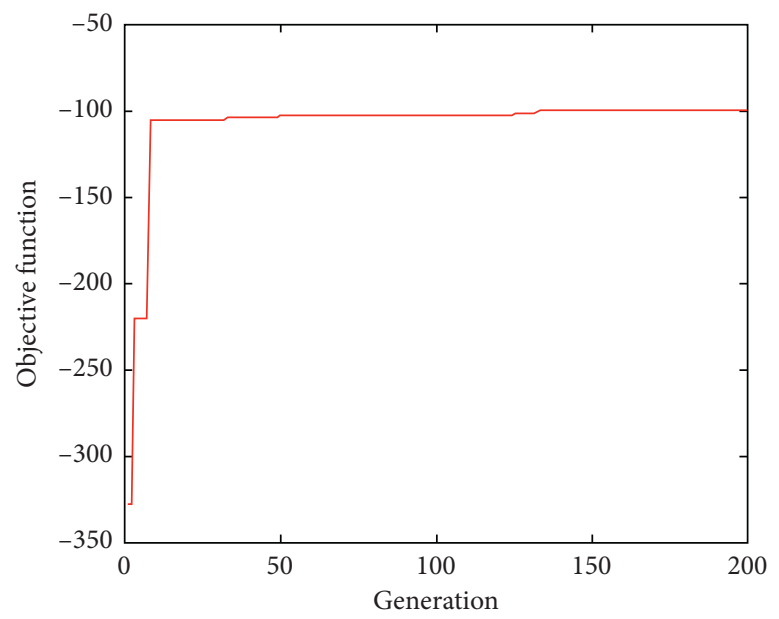

FIGURE 7: Iteration trace of SACG.

TABLE 3: Same results for operation scheduling using different algorithms.

\begin{tabular}{lcccccc}
\hline Method & Successful connections & Successful deliveries & Objective function & OT $\left(t_{\text {sill }}^{h}\right)$ & OD $\left(t_{e_{i}^{\prime l}}^{d}\right)$ & Parallel production lines \\
\hline SA & 10 & 8 & -99.5 & 20.5 & -120 & 3 \\
GA & 10 & 8 & -99.5 & 20.5 & -120 & 3 \\
\hline
\end{tabular}

TABLE 4: Different objective values corresponding to varying SA parameters.

\begin{tabular}{llccccc}
\hline$T_{0} \backslash q$ & 0 & 0.75 & 0.8 & 0.85 & 0.9 & 0.95 \\
\hline 0 & 0 & 0 & 0 & 0 & 0 & 0 \\
100 & 0 & -102 & -102.5 & -99.5 & -99.5 & -99.5 \\
200 & 0 & -101.5 & -99.5 & -99.5 & -99.5 & -99.5 \\
500 & 0 & -99.5 & -99.5 & -99.5 & -99.5 & -99.5 \\
\hline
\end{tabular}

TABLE 5: Results of different objectives.

\begin{tabular}{lccc}
\hline Objective & Successful transfers & Successful deliveries & Objective function \\
\hline Max OT & 10 & 7 & -210 \\
Max OD & 8 & 8 & -104.5 \\
Max (OT + OD) & 10 & 8 & -99.5 \\
\hline
\end{tabular}


TABLE 6: Variation with respect to the production line capacity.

\begin{tabular}{lccccccc}
\hline Capacity & 4 & 6 & 8 & 10 & 15 & 20 & 1000 (Inf) \\
\hline Parallel production lines & 6 & 5 & 4 & 4 & 3 & 3 & -99.5 \\
Objective function & -99.5 & -99.5 & -99.5 & -99.5 & -99.5 & -99.5 \\
Successful connections & 10 & 10 & 10 & 10 & 10 & 10 & 10 \\
Successful deliveries & 8 & 8 & 8 & 8 & 8 & 8 \\
\hline
\end{tabular}

TABLE 7: Changes in objective function values under different costs.

\begin{tabular}{lccccccccccc}
\hline Production line cost & 0 & -10 & -20 & -30 & -40 & -50 & -60 & -70 & -80 & -90 & -100 \\
\hline Production line & 3 & 2 & 2 & 2 & 2 & 2 & 2 & 2 & 2 & 2 & 2 \\
Objective function & -100 & -122 & -141 & -161 & -182 & -203 & -221 & -242 & -262 & -281 & -301 \\
Successful connection & 10 & 10 & 10 & 10 & 10 & 10 & 10 & 10 & 10 & 10 & 10 \\
Successful deliveries & 8 & 8 & 8 & 8 & 8 & 8 & 8 & 8 & 8 & 8 & 8 \\
\hline
\end{tabular}

from equation (3). Taking order 1 as an example, regardless of whether the order selects any trip, the earliest arrival time at its destination is 4.5. The earliest arrival time including the transit time is larger than its delivery time window upper bound. Therefore, variations in the capacity only change the number of parallel production lines and have no effect on delivery success. However, as the capacity gradually increases, the number of parallel production lines will stabilize at a certain value.

In addition, we also tested the effect of the cost change on the function value, and the function value also changes when the cost increases gradually. However, almost the same conclusion is always reached: in the sample system, the number of successful transfers is 10 , and the number of successful deliveries is 8 . The detailed results are shown in Table 7.

\section{Conclusions}

Many studies focus on production allocation, but few pay attention to the coordination and scheduling of orders and transportation in a time-critical mode. This paper focuses on the parallel batch scheduling of production and transportation with respect to synergy in the time dimension. We adopt a scenario analysis method to reveal some internal mechanisms in the transfer and delivery process. Several interconnections between OTR and OTW in the transfer process are found, and OT is introduced based on these findings. Similarly, ODR and ODW in the delivery process are commonly expressed by OD. A column-generationbased simulated annealing algorithm is proposed to solve the problem. Compared with Max OT and Max OD, Max (OT + OD) achieves an obvious improvement in the objective function. Meanwhile, the results of our research are compared with those of similar studies, and the model and algorithm are proven to be workable.

Our research can be further expanded. In practice, due to some unexpected situations, train schedules may change, so it would be worth studying how to formulate strategies to eliminate any negative effects of such changes.

\section{Notations}

$i, i^{\prime}: \quad$ The set of order $i, i^{\prime} \in I ; i$ and $i^{\prime}=1,2, \ldots, k$

$Q_{i}: \quad$ The quantity of order $i$

$p_{i}$ : The processing time of order $i$

$e_{i}$ : The destination of order $i \in I$

$L$ : $\quad$ The set of production lines, $l \in L, L=\{l \mid l=1,2$, $\ldots, n\}$, where $n$ is the total number of production lines

$L^{\prime}: \quad$ The set of trips in the network, $l^{\prime} \in L^{\prime}$, $L^{\prime}=\left\{l^{\prime} \mid l^{\prime}=1,2, \ldots, m\right\}$, where $m$ is the total number of trip indexes

$S\left(l^{\prime}\right): \quad$ The set of stations on trip $l^{\prime}, s \in S\left(l^{\prime}\right)$, $S\left(l^{\prime}\right)=\{s \mid s=1,2, \ldots, p\}$, where $p$ is the total number of stations

$t_{\mathrm{sl}^{\prime}}^{\mathrm{Tra}}$ : The order transfer operating time from $l$ to $l^{\prime}$ at station $s$

$t_{\mathrm{sl}^{\prime}}^{D}: \quad$ The departure time of the last feasible connecting trip at station $s$

$t_{\mathrm{sl}}^{D^{\prime}}: \quad$ The departure time of the selected connecting trip at station $s$; if the selected connecting trip is the last feasible trip, $t_{\mathrm{sl}^{\prime}}^{D^{\prime}}=t_{\mathrm{sl}^{\prime}}^{D}$

$t_{e_{i} l^{\prime}}^{A}$ : The arrival time of trip $l^{\prime}$ at the destination of order $i$

$\left[t_{e_{i}}^{\text {beg }}, t_{e_{i}}^{\text {end }}\right]$ : The delivery time window of order $i$

$t_{\text {él }}^{\text {Tra }}: \quad$ The order delivery operating time from trip $l^{\prime}$ to its destination

$\theta$ : The penalty cost of missing a feasible connecting trip

$\alpha: \quad$ The delivery earliness penalty cost

$\delta: \quad$ The delivery tardiness penalty cost

$\mu: \quad$ The delivery timely contribution benefit

$\mathrm{Cap}_{l^{\prime}}$ : The capacity of trip $l^{\prime}$.

$t_{\mathrm{sil}}^{A}: \quad$ The completion time of order $i$ on production line $l$ at station $s$

$t_{\text {sill' }}^{r}: \quad$ OTR, the time difference between $t_{\mathrm{sl}^{\prime}}^{D}$ and $t_{\text {sil }}^{A}$

$t_{\text {sill' }}^{\text {sill }}$ : OTW, the waiting time for order $i$ when it transfers from production line $l$ to its selected connecting trip $l^{\prime}$

$t_{\text {sill' }}^{h}: \quad$ OT, the order transfer time

ODR, the time difference between $t_{e_{i}}^{\text {end }}$ and $t_{e_{i} l^{\prime}}^{A}$ 
$t_{\boldsymbol{e}_{i} l^{\prime}}^{w}: \quad$ ODW, the waiting time for order $i$ until the delivery is allowed by $t_{e_{i}}^{\text {beg }}$

$t_{e_{i} l^{\prime}}^{d}: \quad$ OD, the order delivery time

$y_{\mathrm{il}^{\prime}}:=1$ if order $i$ selects trip $l^{\prime} ;=0$, otherwise

$r_{i j}^{l}: \quad=1$ if the processing of order $i$ is followed by order $j$ on production line $l ;=0$, otherwise.

\section{Data Availability}

The data used to support the findings of this study are included within the article.

\section{Conflicts of Interest}

The authors declare no conflicts of interest.

\section{Acknowledgments}

This work was supported in part by the National Natural Science Foundation of China (Grant nos. 71801160 and 71601006), the Project from the Department of Education of Liaoning Province (Grant no. WQGD2017024), and the Project of Shenyang Philosophy and Social Science (Grant no. SQ202004L). The authors would like to thank Shenyang University of Technology for offering their Young Teacher Training Program.

\section{References}

[1] J. Shu, C. P. Teo, and Z. J. M. Shen, "Stochastic transportationinventory network design problem," Operations Research, vol. 53, no. 1, pp. 48-60, 2005.

[2] A. Federgruen, G. Prastacos, and P. H. Zipkin, "An allocation and distribution model for perishable products," Operations Research, vol. 34, no. 1, pp. 75-82, 1986.

[3] Z.-L. Chen, "Integrated production and outbound distribution scheduling: review and extensions," Operations Research, vol. 58, no. 1, pp. 130-148, 2010.

[4] Z.-L. Chen and G. L. Vairaktarakis, "Integrated scheduling of production and distribution operations," Management Science, vol. 51, no. 4, pp. 614-628, 2005.

[5] G. Pundoor and Z.-L. Chen, "Scheduling a production-distribution system to optimize the tradeoff between delivery tardiness and distribution cost," Naval Research Logistics, vol. 52, no. 6, pp. 571-589, 2005.

[6] C. Qian, Y. Zhang, Y. Liu, and Z. Wang, "A cloud service platform integrating additive and subtractive manufacturing with high resource efficiency," Journal of Cleaner Production, vol. 241, Article ID 118379, 2019.

[7] W. Jiang, J. Ma, X. Zhang, and H. Xie, "Research on cloud manufacturing resource integrating service modeling based on cloud-Agent," in Proceedings of the 2012 IEEE International Conference on Computer Science and Automation Engineering, pp. 395-398, Beijing, China, 2012.

[8] J.-W. Goossens, S. van Hoesel, and L. Kroon, "On solving multi-type railway line planning problems," European Journal of Operational Research, vol. 168, no. 2, pp. 403-424, 2006.

[9] H. Fu, L. Nie, L. Meng, B. R. Sperry, and Z. He, "A hierarchical line planning approach for a large-scale high speed rail network: the China case," Transportation Research Part A: Policy and Practice, vol. 75, pp. 61-83, 2015.
[10] L. Kang, J. Wu, H. Sun, X. Zhu, and B. Wang, "A practical model for last train rescheduling with train delay in urban railway transit networks," Omega, vol. 50, pp. 29-42, 2015.

[11] X. Wu, L. Nie, and M. Xu, "Designing an integrated distribution system for catering services for high-speed railways: a three-echelon location routing model with tight time windows and time deadlines," Transportation Research Part C: Emerging Technologies, vol. 74, pp. 212-244, 2017.

[12] M. Díaz-Madroñero, D. Peidro, and J. Mula, "A review of tactical optimization models for integrated production and transport routing planning decisions," Computers \& Industrial Engineering, vol. 88, pp. 518-535, 2015.

[13] L. S. Cao, J. H. Hao, and D. K. Jiang, "Two parallel machines scheduling with two-vehicle job delivery to minimize makespan," Complexity, vol. 2020, Article ID 1647401, 6 pages, 2020.

[14] C. Pornsing, P. Tharawetcharak, P. Jomthong, and T. Tonglim, "Particle swarm optimization for integrated production-distribution scheduling problem," in Proceedings of the 2017 3rd International Conference on Control, Automation and Robotics (ICCAR), Nagoya, Japan, April 2017.

[15] X. Zhong and D. Jiang, "Integrated scheduling of production and distribution with release dates and capacitated deliveries," Mathematical Problem in Engineering, vol. 2016, Article ID 9315197, 5 pages, 2016.

[16] R. Russel, W. C. Chiang, and D. Zepeda, "Integrating multiproduct production and distribution in newspaper logistics," Computers \& Operations Research, vol. 35, pp. 1576-1588, 2008.

[17] T. Kishimoto, K. Fugo, and T. Kiyokawa, "Integrated production and intermodal transportation planning in large scale production-distribution-networks," Transportation Research, vol. 60, pp. 62-78, 2013.

[18] Y. Ma, F. Yan, K. Kang, and X. Wei, “A novel integrated production-distribution planning model with conflict and coordination in a supply chain network," Knowledge-Based Systems, vol. 105, pp. 119-133, 2016.

[19] M. T. Melo, S. Nickel, and F. Saldanha-da-Gama, "Facility location and supply chain management - a review," European Journal of Operational Research, vol. 196, no. 2, pp. 401-412, 2009.

[20] Y.-C. Chang and C.-Y. Lee, "Machine scheduling with job delivery coordination," European Journal of Operational Research, vol. 158, no. 2, pp. 470-487, 2004.

[21] M. Hajiaghaei-Keshteli, M. Aminnayeri, and S. M. T. Fatemi Ghomi, "Integrated scheduling of production and rail transportation," Computers \& Industrial Engineering, vol. 74, pp. 240-256, 2014.

[22] C.-Y. Lee and Z.-L. Chen, "Machine scheduling with transportation considerations," Journal of Scheduling, vol. 4, no. 1, pp. 3-24, 2001.

[23] A. Soukhal, A. Oulamara, and P. Martineau, "Complexity of flow shop scheduling problems with transportation constraints," European Journal of Operational Research, vol. 161, no. 1, pp. 32-41, 2005.

[24] L. Lu, J. Yuan, and L. Zhang, "Single machine scheduling with release dates and job delivery to minimize the makespan," Theoretical Computer Science, vol. 393, no. 1-3, pp. 102-108, 2008.

[25] L. Tang and H. Gong, "The coordination of transportation and batching scheduling," Applied Mathematical Modelling, vol. 33, no. 10, pp. 3854-3862, 2009.

[26] S. Bock, "Finding optimal tour schedules on transportation paths under extended time window constraints," Journal of Scheduling, vol. 19, pp. 1-20, 2016. 
[27] V. Abdollahzadeh, I. Nakhaikamalabadi, S. M. Hajimolana, and S. H. Zegordi, "A multifactory integrated production and distribution scheduling problem with parallel machines and immediate shipments solved by improved whale optimization algorithm," Complexity, pp. 1-21, 2018.

[28] B.-Y. Cheng, J. Y.-T. Leung, and K. Li, "Integrated scheduling of production and distribution to minimize total cost using an improved ant colony optimization method," Computers \& Industrial Engineering, vol. 83, pp. 217-225, 2015.

[29] P. Devapriya, W. Ferrell, and N. Geismar, "Integrated production and distribution scheduling with a perishable product," European Journal of Operational Research, vol. 259, no. 3, pp. 906-916, 2017.

[30] A. Noroozi, M. M. Mazdeh, M. Heydari, and M. Rasti-Barzoki, "Coordinating order acceptance and integrated production-distribution scheduling with batch delivery considering third party logistics distribution," Journal of Manufacturing Systems, vol. 46, pp. 29-45, 2018.

[31] Y. Jiang, X. Zhou, and Q. Xu, "Scenario analysis-based decision and coordination in supply chain management with production and transportation scheduling," Symmetry, vol. 11, no. 2, p. 160, 2019.

[32] S. Moons, K. Ramaekers, C. An, and Y. Arda, "Integrating production scheduling and vehicle routing decisions at the operational decision level: a review and discussion," Computers and Industrial Engineering, vol. 104, pp. 224-245, 2016.

[33] X. Wang and T. C. E. Cheng, "Machine scheduling with an availability constraint and job delivery coordination," Naval Research Logistics, vol. 54, no. 1, pp. 11-20, 2007.

[34] H. Xuan, "Hybrid flowshop scheduling with finite transportation capacity," Applied Mechanics and Materials, vol. 65, pp. 574-578, 2011.

[35] W. Zhong, G. Dósa, and Z. Tan, "On the machine scheduling problem with job delivery coordination," European Journal of Operational Research, vol. 182, pp. 1057-1072, 2015.

[36] S. M. Seyedhosseini and S. M. Ghoreyshi, "An integrated model for production and distribution planning of perishable products with inventory and routing considerations," Mathematical Problems in Engineering, vol. 2014, pp. 8-10, Article ID 475606, 2014.

[37] H. Gong, D. Chen, and K. Xu, "Parallel-batch scheduling and transportation coordination with waiting time constraint," The Scientific World Journal, vol. 2014, 8 pages, 2014.

[38] M. Zandieh and S. Molla-Alizadeh-Zavardehi, Synchronizing Production and Air Transportation Scheduling Using Mathematical Programming Models, Elsevier Science Publishers, Amsterdam, The Netherlands, 2009.

[39] M. R. Delavar, M. Hajiaghaei-Keshteli, and S. Molla-Alizadeh-Zavardehi, "Genetic algorithms for coordinated scheduling of production and air transportation," Expert Systems with Applications, vol. 37, pp. 8255-8266, 2010.

[40] F. Azadian, A. Murat, and R. B. Chinnam, "Integrated production and logistics planning: contract manufacturing and choice of air/surface transportation," European Journal of Operational Research, vol. 247, no. 1, pp. 113-123, 2015.

[41] R. G. Pemberton, "A lotus 1-2-3 model for airline level of operations planning," Computers \& Industrial Engineering, vol. 11, no. 1-4, pp. 330-334, 1986.

[42] J. Perl and M. S. Daskin, "A warehouse location-routing problem," Transportation Research Part B: Methodological, vol. 19, no. 5, pp. 381-396, 1985.

[43] G. Nagy and S. Salhi, "Location-routing: issues, models and methods," European Journal of Operational Research, vol. 177, no. 2, pp. 649-672, 2007.
[44] H. F. Du, J. R. Fan, X. C. He, and M. W. Feldman, "A genetic simulated annealing algorithm to optimize the small-world network generating process," Complexity, vol. 2018, Article ID 1453898, 12 pages, 2018.

[45] S. W. Lin, K. C. Ying, Z. J. Lee, and H. S. Chen, "Vehicle routing problems with time windows using simulated annealing," IEEE International Conference on Systems, Man and Cybernetics, 2006.

[46] M. Hamzeei, R. Z. Farahani, and H. Rashidi-Bejgan, "An exact and a simulated annealing algorithm for simultaneously determining flow path and the location of $\mathrm{P} / \mathrm{D}$ stations in bidirectional path," Journal of Manufacturing Systems, vol. 32, no. 4, pp. 648-654, 2013.

[47] J. R. Daduna and S. Vo $\beta$, "Practical experiences in schedule synchronization," In J. R. Daduna, I. Branco, J. M. P. Paixão (eds) Computer-Aided Transit Scheduling. Lecture Notes in Economics and Mathematical Systems, vol. 430, pp. 39-55, Springer, Berlin, Germany, 1995, https://doi.org/10.1007/9783-642-57762-8_4.

[48] T. L. Friesz, H.-J. Cho, N. J. Mehta, R. L. Tobin, and G. Anandalingam, "A simulated annealing approach to the network design problem with variational inequality constraints," Transportation Science, vol. 26, no. 1, pp. 18-26, 1992.

[49] Y. Wei and L. Zhou, "Soft time windows associated vehicles routing problems of logistics distribution center using genetic simulated annealing algorithm," Journal of Computing and Information Technology, vol. 22, no. LISS 2013, p. 31, 2014.

[50] R. Ahin and O. Türkbey, "A simulated annealing algorithm to find approximate Pareto optimal solutions for the multi-objective facility layout problem," The International Journal of Advanced Manufacturing Technology, vol. 41, pp. 1003-1018, 2009.

[51] M. D. Soumis, "A column generation approach to the urban transit crew scheduling problem," Transportation Science, vol. 23, pp. 1-13, 1989.

[52] Z.-L. Chen and W. B. Powell, "A column generation based decomposition algorithm for a parallel machine just-in-time scheduling problem," European Journal of Operational Research, vol. 116, no. 1, pp. 220-232, 1999.

[53] R. A. Skitt and R. R. Levary, "Vehicle routing via column generation," European Journal of Operational Research, vol. 21, no. 1, pp. 65-76, 1985.

[54] Z. Liang, F. Xiao, X. Qian et al., “A column generation-based heuristic for aircraft recovery problem with airport capacity constraints and maintenance flexibility," Transportation Research Part B: Methodological, vol. 113, pp. 70-90, 2018.

[55] Y. Wang, J. Tang, and R. Y. K. Fung, "A column-generationbased heuristic algorithm for solving operating theater planning problem under stochastic demand and surgery cancellation risk," International Journal of Production Economics, vol. 158, pp. 28-36, 2014. 